\title{
A $q$-Analogue of Faulhaber's Formula for Sums of Powers
}

\author{
Victor J. W. Guo and Jiang Zeng \\ Institut Camille Jordan, Université Claude Bernard (Lyon I) \\ F-69622 Villeurbanne Cedex, France \\ jwguo@eyou.com, zeng@math.univ-lyon1.fr
}

Submitted: Jan 25, 2005; Accepted: Aug 16, 2005; Published: Aug 30, 2005

Mathematics Subject Classifications: 05A30, 05A15

Dedicated to Richard Stanley on the occasion of his 60th birthday

\section{Abstract}

Let

$$
S_{m, n}(q):=\sum_{k=1}^{n} \frac{1-q^{2 k}}{1-q^{2}}\left(\frac{1-q^{k}}{1-q}\right)^{m-1} q^{\frac{m+1}{2}(n-k)} .
$$

Generalizing the formulas of Warnaar and Schlosser, we prove that there exist polynomials $P_{m, k}(q) \in \mathbb{Z}[q]$ such that

$$
S_{2 m+1, n}(q)=\sum_{k=0}^{m}(-1)^{k} P_{m, k}(q) \frac{\left(1-q^{n}\right)^{m+1-k}\left(1-q^{n+1}\right)^{m+1-k} q^{k n}}{\left(1-q^{2}\right)(1-q)^{2 m-3 k} \prod_{i=0}^{k}\left(1-q^{m+1-i}\right)},
$$

and solve a problem raised by Schlosser. We also show that there is a similar formula for the following $q$-analogue of alternating sums of powers:

$$
T_{m, n}(q):=\sum_{k=1}^{n}(-1)^{n-k}\left(\frac{1-q^{k}}{1-q}\right)^{m} q^{\frac{m}{2}(n-k)} .
$$

\section{Introduction}

In the early 17th century Faulhaber [1] computed the sums of powers $1^{m}+2^{m}+\cdots+n^{m}$ up to $m=17$ and realized that for odd $m$, it is not just a polynomial in $n$ but a polynomial in the triangular number $N=n(n+1) / 2$. A good account of Faulhaber's work was given by Knuth [7]. For example, for $m=1, \ldots, 5$, Faulhaber's formulas read as follows:

$$
\begin{aligned}
& 1^{1}+2^{1}+\cdots+n^{1}=N, \quad N=\left(n^{2}+n\right) / 2 ; \\
& 1^{2}+2^{2}+\cdots+n^{2}=\frac{2 n+1}{3} N ;
\end{aligned}
$$




$$
\begin{aligned}
& 1^{3}+2^{3}+\cdots+n^{3}=N^{2} \\
& 1^{4}+2^{4}+\cdots+n^{4}=\frac{2 n+1}{5}\left(2 N^{2}-\frac{1}{3} N\right) ; \\
& 1^{5}+2^{5}+\cdots+n^{5}=\frac{1}{3}\left(4 N^{3}-N^{2}\right) .
\end{aligned}
$$

Recently, the problem of $q$-analogues of the sums of powers has attracted the attention of several authors $[2,9,8]$, who found, in particular, $q$-analogues of the Faulhaber formula corresponding to $m=1,2, \ldots, 5$. More precisely, setting

$$
S_{m, n}(q)=\sum_{k=1}^{n} \frac{1-q^{2 k}}{1-q^{2}}\left(\frac{1-q^{k}}{1-q}\right)^{m-1} q^{\frac{m+1}{2}(n-k)}
$$

Warnaar [9] (for $m=3$ ) and Schlosser [8] found the following formulas for the $q$-analogues of the sums of consecutive integers, squares, cubes, quarts and quints:

$$
\begin{aligned}
& S_{1, n}(q)=\frac{\left(1-q^{n}\right)\left(1-q^{n+1}\right)}{(1-q)\left(1-q^{2}\right)}, \\
& S_{2, n}(q)=\frac{\left(1-q^{n}\right)\left(1-q^{n+1}\right)\left(1-q^{n+\frac{1}{2}}\right)}{(1-q)\left(1-q^{2}\right)\left(1-q^{\frac{3}{2}}\right)} \\
& S_{3, n}(q)=\frac{\left(1-q^{n}\right)^{2}\left(1-q^{n+1}\right)^{2}}{(1-q)^{2}\left(1-q^{2}\right)^{2}}, \\
& S_{4, n}(q)=\frac{\left(1-q^{n}\right)\left(1-q^{n+1}\right)\left(1-q^{n+\frac{1}{2}}\right)}{(1-q)\left(1-q^{2}\right)\left(1-q^{\frac{5}{2}}\right)}\left[\frac{\left(1-q^{n}\right)\left(1-q^{n+1}\right)}{(1-q)^{2}}-\frac{1-q^{\frac{1}{2}}}{1-q^{\frac{3}{2}}} q^{n}\right], \\
& S_{5, n}(q)=\frac{\left(1-q^{n}\right)^{2}\left(1-q^{n+1}\right)^{2}}{(1-q)^{2}\left(1-q^{2}\right)\left(1-q^{3}\right)}\left[\frac{\left(1-q^{n}\right)\left(1-q^{n+1}\right)}{(1-q)^{2}}-\frac{1-q}{1-q^{2}} q^{n}\right] .
\end{aligned}
$$

Notice that the above formulas have the same pattern that each summand on the righthand side has no pole at $q=1$, and so reduce directly to Faulhaber's corresponding formulas when $q \rightarrow 1$.

At the end of his paper, Schlosser [8] speculated on the existence of a general formula for $S_{m, n}(q)$, and left it as an open problem. It is the purpose of this paper to provide such a general formula, which turns out to be a $q$-analogue of the Faulhaber formula for the sums of powers. More precisely, we prove the following results:

Theorem 1.1 For $m, n \in \mathbb{N}$, there exist polynomials $P_{m, k}(q) \in \mathbb{Z}[q]$ such that

$$
S_{2 m+1, n}(q)=\sum_{k=0}^{m}(-1)^{k} P_{m, k}(q) \frac{\left(1-q^{n}\right)^{m+1-k}\left(1-q^{n+1}\right)^{m+1-k} q^{k n}}{\left(1-q^{2}\right)(1-q)^{2 m-3 k} \prod_{i=0}^{k}\left(1-q^{m+1-i}\right)}
$$


where $P_{m, s}(q)$ are the $q$-Faulhaber coefficients given by

$$
\begin{aligned}
P_{m, s}(q)= & \frac{\prod_{j=0}^{s}\left(1-q^{m+1-j}\right)}{(1-q)^{3 s}} \sum_{k=0}^{s} \frac{(-1)^{s-k}}{1-q^{m+1-k}}\left[\left(\begin{array}{c}
2 m \\
k
\end{array}\right)-\left(\begin{array}{c}
2 m \\
k-2
\end{array}\right)\right] \\
& \times \sum_{i=0}^{s-k} \frac{m-k+1}{m-s+1}\left(\begin{array}{c}
m-s+i \\
i
\end{array}\right)\left(\begin{array}{c}
m-k-i \\
s-k-i
\end{array}\right) q^{s-k-i} .
\end{aligned}
$$

Theorem 1.2 For $m, n \in \mathbb{N}$, there exist polynomials $Q_{m, k}(q) \in \mathbb{Z}[q]$ such that

$$
S_{2 m, n}(q)=\sum_{k=0}^{m}(-1)^{k} Q_{m, k}\left(q^{\frac{1}{2}}\right) \frac{\left(1-q^{n+\frac{1}{2}}\right)\left(1-q^{n}\right)^{m-k}\left(1-q^{n+1}\right)^{m-k}\left(1-q^{\frac{1}{2}}\right)^{k} q^{k n}}{\left(1-q^{2}\right)(1-q)^{2 m-2 k-1} \prod_{i=0}^{k}\left(1-q^{m-i+\frac{1}{2}}\right)} .
$$

Furthermore, we have

$$
\begin{aligned}
Q_{m, s}(q)= & \frac{\prod_{j=0}^{s}\left(1-q^{2 m-2 j+1}\right)}{(1-q)^{s}\left(1-q^{2}\right)^{2 s}} \sum_{k=0}^{s} \frac{(-1)^{s-k}}{1-q^{2 m-2 k+1}}\left[\left(\begin{array}{c}
2 m-1 \\
k
\end{array}\right)-\left(\begin{array}{c}
2 m-1 \\
k-2
\end{array}\right)\right] \\
& \times \sum_{i=0}^{s-k}\left(\begin{array}{c}
m-s+i \\
i
\end{array}\right)\left[\left(\begin{array}{c}
m-k-i \\
s-k-i
\end{array}\right) q^{2 s-2 k-2 i}+\left(\begin{array}{c}
m-k-i-1 \\
s-k-i-1
\end{array}\right) q^{2 s-2 k-2 i-1}\right] .
\end{aligned}
$$

Next we consider a $q$-analogue of the alternating sums $T_{m, n}=\sum_{k=1}^{n}(-1)^{n-k} k^{m}$. Note that Gessel and Viennot [4] proved that $T_{2 m, n}$ can be written as a polynomial in $n(n+1)$ whose coefficients are the Salié coefficients and Schlosser [8] gave some $q$-analogues of $T_{m, n}$ only for $m \leq 4$. Let

$$
T_{m, n}(q)=\sum_{k=1}^{n}(-1)^{n-k}\left(\frac{1-q^{k}}{1-q}\right)^{m} q^{\frac{m}{2}(n-k)} .
$$

We have the following $q$-analogue of Gessel-Viennot's result for $T_{m, n}$.

Theorem 1.3 For $m, n \in \mathbb{N}$, there exist polynomials $G_{m, k}(q) \in \mathbb{Z}[q]$ such that

$$
T_{2 m, n}(q)=\sum_{k=0}^{m-1}(-1)^{k} G_{m, k}(q) \frac{\left(1-q^{n}\right)^{m-k}\left(1-q^{n+1}\right)^{m-k} q^{k n}}{(1-q)^{2 m-2 k} \prod_{i=0}^{k}\left(1+q^{m-i}\right)}
$$

where $G_{m, k}(q)$ are the q-Salié coefficients given by

$$
\begin{aligned}
G_{m, s}(q)= & \frac{\prod_{j=0}^{s}\left(1+q^{m-j}\right)}{(1-q)^{2 s}} \sum_{k=0}^{s} \frac{(-1)^{s-k}}{1+q^{m-k}}\left(\begin{array}{c}
2 m \\
k
\end{array}\right) \\
& \times \sum_{i=0}^{s-k} \frac{m-k}{m-s}\left(\begin{array}{c}
m-s+i-1 \\
i
\end{array}\right)\left(\begin{array}{c}
m-k-i-1 \\
s-k-i
\end{array}\right) q^{s-k-i} .
\end{aligned}
$$


Theorem 1.4 For $m, n \in \mathbb{N}$, there exist polynomials $H_{m, k}(q) \in \mathbb{Z}[q]$ such that

$$
\begin{aligned}
T_{2 m-1, n}(q)= & (-1)^{m+n} H_{m, m-1}\left(q^{\frac{1}{2}}\right) \frac{q^{m n-\frac{n}{2}}}{\left(1+q^{\frac{1}{2}}\right)^{m} \prod_{i=0}^{m-1}\left(1+q^{m-i-\frac{1}{2}}\right)} \\
& +\frac{1-q^{n+\frac{1}{2}}}{1-q^{\frac{1}{2}}} \sum_{k=0}^{m-1}(-1)^{k} \frac{H_{m, k}\left(q^{\frac{1}{2}}\right)\left(1-q^{n}\right)^{m-k-1}\left(1-q^{n+1}\right)^{m-k-1} q^{k n}}{(1-q)^{2 m-2 k-2}\left(1+q^{\frac{1}{2}}\right)^{k+1} \prod_{i=0}^{k}\left(1+q^{m-i-\frac{1}{2}}\right)} .
\end{aligned}
$$

Furthermore, we have

$$
\begin{aligned}
H_{m, s}(q)= & \frac{\prod_{j=0}^{s}\left(1+q^{2 m-2 j-1}\right)}{(1+q)^{s}(1-q)^{2 s}} \sum_{k=0}^{s} \frac{(-1)^{s-k}}{1+q^{2 m-2 k-1}}\left(\begin{array}{c}
2 m-1 \\
k
\end{array}\right) \sum_{i=0}^{s-k}\left(\begin{array}{c}
m-s+i-1 \\
i
\end{array}\right) \\
& \times\left[\left(\begin{array}{c}
m-k-i-1 \\
s-k-i
\end{array}\right) q^{2 s-2 k-2 i}+\left(\begin{array}{c}
m-k-i-2 \\
s-k-i-1
\end{array}\right) q^{2 s-2 k-2 i-1}\right] .
\end{aligned}
$$

Schlosser [8] derives his formulas from the machinery of basic hypergeometric series. For example, for the $q$-analogues of the sums of quarts and quints, he first specializes Bailey's terminating very-well-poised balanced ${ }_{10} \phi_{9}$ transformation [3, Appendix (III.28)] and then applies the terminating very-well-poised ${ }_{6} \phi_{5}[3$, Appendix (II.21)] on one side of the identity to establish a "master identity." In contrast to his proof, our method is self-contained and of elementary nature.

We first establish some elementary algebraic identities in Section 2, and prove Theorems 1.1-1.4 in Section 3. We then apply our theorems to compute the polynomials $P_{m, s}(q), Q_{m, s}(q), G_{m, s}(q)$, and $H_{m, s}(q)$ for small $m$ in Section 4 and obtain summation formulas of $(1.1)$ for $m \leq 11$. Section 5 contains some further extensions of these summation formulas.

\section{Some Preliminary Lemmas}

The following is our first step towards our summation formula for $S_{m, n}(q)$.

Lemma 2.1 For $m, n \in \mathbb{N}$, we have

$$
S_{m, n}(q)=\sum_{r=0}^{\left\lfloor\frac{m}{2}\right\rfloor}(-1)^{r}\left[\left(\begin{array}{c}
m-1 \\
r
\end{array}\right)-\left(\begin{array}{c}
m-1 \\
r-2
\end{array}\right)\right] \frac{\left(1-q^{\left(\frac{m+1}{2}-r\right) n}\right)\left(1+(-1)^{m} q^{\left(\frac{m+1}{2}-r\right)(n+1)}\right) q^{r n}}{\left(1-q^{2}\right)(1-q)^{m-1}\left(1-q^{\frac{m+1}{2}-r}\right)} .
$$

Proof. By definition, $\left(1-q^{2}\right)(1-q)^{m-1} S_{m, n}(q)$ is equal to

$$
\begin{aligned}
& \sum_{k=1}^{n}\left(1-q^{2 k}\right)\left(1-q^{k}\right)^{m-1} q^{\frac{m+1}{2}(n-k)} \\
& \quad=\sum_{k=1}^{n}\left(1-q^{2 k}\right) q^{\frac{m+1}{2}(n-k)} \sum_{r=0}^{m-1}\left(\begin{array}{c}
m-1 \\
r
\end{array}\right)(-1)^{r} q^{k r}
\end{aligned}
$$




$$
\begin{aligned}
& =\sum_{r=0}^{m-1}\left(\begin{array}{c}
m-1 \\
r
\end{array}\right)(-1)^{r} \sum_{k=1}^{n}\left(q^{\frac{m+1}{2} n+\left(r-\frac{m+1}{2}\right) k}-q^{\frac{m+1}{2} n+\left(r-\frac{m-3}{2}\right) k}\right) \\
& =\sum_{r=0}^{m+1}(-1)^{r}\left[\left(\begin{array}{c}
m-1 \\
r
\end{array}\right)-\left(\begin{array}{c}
m-1 \\
r-2
\end{array}\right)\right] \sum_{k=1}^{n} q^{\frac{m+1}{2} n+\left(r-\frac{m+1}{2}\right) k} \\
& =\sum_{\substack{r=0 \\
r \neq \frac{m+1}{2}}}^{m+1}(-1)^{r}\left[\left(\begin{array}{c}
m-1 \\
r
\end{array}\right)-\left(\begin{array}{c}
m-1 \\
r-2
\end{array}\right)\right] \frac{q^{\frac{m+1}{2}(n-1)+r}-q^{r(n+1)-\frac{m+1}{2}}}{1-q^{r-\frac{m+1}{2}}} .
\end{aligned}
$$

Splitting the last summation into two parts corresponding to $r$ ranging from 0 to $\left\lfloor\frac{m}{2}\right\rfloor$ and from $\left\lfloor\frac{m+1}{2}\right\rfloor+1$ to $m+1$, respectively. Replacing $r$ by $m+1-r$ in the second one we can rewrite $(2.2)$ as follows:

$$
\begin{aligned}
& \sum_{r=0}^{\left\lfloor\frac{m}{2}\right\rfloor}(-1)^{r}\left[\left(\begin{array}{c}
m-1 \\
r
\end{array}\right)-\left(\begin{array}{c}
m-1 \\
r-2
\end{array}\right)\right] \\
& \quad \times\left[\frac{q^{\frac{m+1}{2}(n-1)+r}-q^{r(n+1)-\frac{m+1}{2}}}{1-q^{r-\frac{m+1}{2}}}+(-1)^{m} \frac{q^{\frac{m+1}{2}(n+1)-r}-q^{\frac{m+1}{2}(2 n+1)-r(n+1)}}{1-q^{\frac{m+1}{2}-r}}\right] .
\end{aligned}
$$

After simplification we get (2.1).

Remark. When $m$ is even, since

$$
\sum_{r=0}^{\frac{m}{2}}(-1)^{r}\left[\left(\begin{array}{c}
m-1 \\
r
\end{array}\right)-\left(\begin{array}{c}
m-1 \\
r-2
\end{array}\right)\right]=0
$$

we can rewrite $S_{m, n}(q)$ as

$$
S_{m, n}(q)=\sum_{r=0}^{\frac{m}{2}}(-1)^{r}\left[\left(\begin{array}{c}
m-1 \\
r
\end{array}\right)-\left(\begin{array}{c}
m-1 \\
r-2
\end{array}\right)\right] \frac{\left(1-q^{\left(\frac{m+1}{2}-r\right)(2 n+1)}\right) q^{n r}}{\left(1-q^{2}\right)(1-q)^{m-1}\left(1-q^{\frac{m+1}{2}-r}\right)}
$$

Lemma 2.2 For $m, n \geq 1$, we have

$$
T_{2 m, n}(q)=\sum_{r=0}^{m-1}(-1)^{r}\left(\begin{array}{c}
2 m \\
r
\end{array}\right) \frac{\left(1-q^{n(m-r)}\right)\left(1-q^{(n+1)(m-r)}\right) q^{r n}}{(1-q)^{2 m}\left(1+q^{m-r}\right)} .
$$

Proof. By (1.9) we have

$$
(1-q)^{2 m} T_{2 m, n}(q)=\sum_{k=1}^{n}\left(1-q^{k}\right)^{2 m} q^{m(n-k)}(-1)^{n-k}
$$


Expanding $\left(1-q^{k}\right)^{2 m}$ by the binomial theorem and exchanging the summation order, we obtain

$$
(1-q)^{2 m} T_{2 m, n}(q)=\sum_{r=0}^{2 m}(-1)^{r}\left(\begin{array}{c}
2 m \\
r
\end{array}\right) q^{r n} \frac{1-\left(-q^{m-r}\right)^{n}}{1+q^{m-r}} .
$$

Substituting $r$ by $2 m-r$ on the right-hand side of (2.5) yields

$$
\begin{aligned}
(1-q)^{2 m} T_{2 m, n}(q) & =\frac{1}{2} \sum_{r=0}^{2 m}(-1)^{r}\left(\begin{array}{c}
2 m \\
r
\end{array}\right)\left[q^{r n} \frac{1-\left(-q^{m-r}\right)^{n}}{1+q^{m-r}}+q^{(2 m-r) n} \frac{1-\left(-q^{r-m}\right)^{n}}{1+q^{r-m}}\right] \\
& =\frac{1}{2} \sum_{r=0}^{2 m}(-1)^{r}\left(\begin{array}{c}
2 m \\
r
\end{array}\right) q^{r n} \frac{\left(1-(-1)^{n} q^{n(m-r)}\right)\left(1-(-1)^{n} q^{(n+1)(m-r)}\right)}{1+q^{m-r}} \\
& =\frac{1}{2} \sum_{r=0}^{2 m}(-1)^{r}\left(\begin{array}{c}
2 m \\
r
\end{array}\right) q^{r n} \frac{\left(1-q^{n(m-r)}\right)\left(1-q^{(n+1)(m-r)}\right)}{1+q^{m-r}}
\end{aligned}
$$

The last equality holds because

$$
\sum_{r=0}^{2 m}(-1)^{r}\left(\begin{array}{c}
2 m \\
r
\end{array}\right) q^{r n} \frac{q^{n(m-r)}+q^{(n+1)(m-r)}}{1+q^{m-r}}=0 .
$$

Splitting the sum in (2.6) as $\sum_{r=0}^{m-1}+\sum_{r=m+1}^{2 m}$ and substituting $r$ by $2 m-r$ in the second sum, we complete the proof.

Similarly, we can show that $2(1-q)^{2 m-1} T_{2 m-1, n}(q)$ is equal to

$$
\begin{aligned}
& \sum_{r=0}^{2 m-1}(-1)^{r}\left(\begin{array}{c}
2 m-1 \\
r
\end{array}\right) q^{r n} \frac{\left(1-(-1)^{n} q^{n\left(m-r-\frac{1}{2}\right)}\right)\left(1+(-1)^{n} q^{(n+1)\left(m-r-\frac{1}{2}\right)}\right)}{1+q^{m-r-\frac{1}{2}}} \\
& \quad=\sum_{r=0}^{2 m-1}(-1)^{r}\left(\begin{array}{c}
2 m-1 \\
r
\end{array}\right)\left[(-1)^{n+1} q^{\left(m-\frac{1}{2}\right) n} \frac{1-q^{m-r-\frac{1}{2}}}{1+q^{m-r-\frac{1}{2}}}+q^{r n} \frac{1-q^{(2 n+1)\left(m-r-\frac{1}{2}\right)}}{1+q^{m-r-\frac{1}{2}}}\right] .
\end{aligned}
$$

This establishes immediately the following lemma:

Lemma 2.3 For $m, n \geq 1$, we have

$$
\begin{aligned}
T_{2 m-1, n}(q)= & \sum_{r=0}^{m-1}(-1)^{n+r+1}\left(\begin{array}{c}
2 m-1 \\
r
\end{array}\right) \frac{\left(1-q^{m-r-\frac{1}{2}}\right) q^{\left(m-\frac{1}{2}\right) n}}{(1-q)^{2 m-1}\left(1+q^{m-r-\frac{1}{2}}\right)} \\
& +\sum_{r=0}^{m-1}(-1)^{r}\left(\begin{array}{c}
2 m-1 \\
r
\end{array}\right) \frac{\left(1-q^{(2 n+1)\left(m-r-\frac{1}{2}\right)}\right) q^{r n}}{(1-q)^{2 m-1}\left(1+q^{m-r-\frac{1}{2}}\right)} .
\end{aligned}
$$

The second ingredient of our approach is the following identity, of which we shall give two proofs. 
Theorem 2.4 For $m \in \mathbb{N}$, we have

$$
\frac{1-x^{m+1} y^{m+1}}{(1-x y)(1-x)^{m}(1-y)^{m}}=\sum_{r=0}^{m} \sum_{s=o}^{m-r}\left(\begin{array}{c}
m-r \\
s
\end{array}\right)\left(\begin{array}{c}
m-s \\
r
\end{array}\right) \frac{x^{r} y^{s}}{(1-x)^{r+s}(1-y)^{r+s}} .
$$

First Proof. Replacing $s$ by $m-r-s$, the right-hand side of (2.8) may be written as

$$
\sum_{r=0}^{m} \sum_{s=o}^{m-r}\left(\begin{array}{c}
m-r \\
s
\end{array}\right)\left(\begin{array}{c}
r+s \\
r
\end{array}\right) \frac{x^{r} y^{m-r-s}}{(1-x)^{m-s}(1-y)^{m-s}}
$$

Consider the generating function of (2.9). We have

$$
\begin{aligned}
& \sum_{m=0}^{\infty} \sum_{r=0}^{m} \sum_{s=o}^{m-r}\left(\begin{array}{c}
m-r \\
s
\end{array}\right)\left(\begin{array}{c}
r+s \\
r
\end{array}\right) \frac{x^{r} y^{m-r-s}}{(1-x)^{m-s}(1-y)^{m-s}} t^{m} \\
& =\sum_{r=0}^{\infty} \sum_{s=0}^{\infty}\left(\begin{array}{c}
r+s \\
r
\end{array}\right) x^{r} \sum_{m=r+s}^{\infty}\left(\begin{array}{c}
m-r \\
s
\end{array}\right) \frac{y^{m-r-s}}{(1-x)^{m-s}(1-y)^{m-s}} t^{m} \\
& =\sum_{r=0}^{\infty} \sum_{s=0}^{\infty}\left(\begin{array}{c}
r+s \\
r
\end{array}\right) \frac{x^{r} t^{r+s}}{(1-x)^{r}(1-y)^{r}}\left(1-\frac{y t}{(1-x)(1-y)}\right)^{-s-1} \\
& =\frac{(1-x)(1-y)}{(1-x)(1-y)-y t}\left(1-\frac{x t}{(1-x)(1-y)}-\frac{t(1-x)(1-y)}{(1-x)(1-y)-y t}\right)^{-1} \\
& =\frac{[(1-x)(1-y)-x y t][(1-x)(1-y)-t]}{[1-y)^{2}}
\end{aligned}
$$

which is equal to the generating function of the left-hand side of (2.8).

Second Proof. Let

$$
\left\{\begin{array}{l}
x=u(1-x)(1-y) \\
y=v(1-x)(1-y)
\end{array}\right.
$$

We want to expand

$$
f(x, y)=\frac{1-x^{m+1} y^{m+1}}{(1-x y)(1-x)^{m}(1-y)^{m}}
$$

as a series in $u$ and $v$. By Lagrange's inversion formula (see, for example, [5, p. 21]),

$$
f(x, y)=\sum_{r, s \geq 0} u^{r} v^{s}\left[x^{r} y^{s}\right]\left\{\frac{1-x^{m+1} y^{m+1}}{(1-x y)(1-x)^{m-r-s}(1-y)^{m-r-s}} \Delta\right\},
$$

where $\left[x^{r} y^{s}\right] F(x, y)$ denotes the coefficient of $x^{r} y^{s}$ in the power series $F(x, y)$, and where $\Delta$ is the determinant given by

$$
\Delta=\left|\begin{array}{rr}
1+\frac{x}{1-x} & \frac{y}{1-y} \\
\frac{x}{1-x} & 1+\frac{y}{1-y}
\end{array}\right|=\frac{1-x y}{(1-x)(1-y)} .
$$


So,

$$
f(x, y)=\sum_{r, s \geq 0} u^{r} v^{s}\left[x^{r} y^{s}\right]\left\{\frac{1-x^{m+1} y^{m+1}}{(1-x)^{m-r-s+1}(1-y)^{m-r-s+1}}\right\}
$$

Since

$$
(1-z)^{-\alpha}=\sum_{k=0}^{\infty}\left(\begin{array}{c}
\alpha+k-1 \\
k
\end{array}\right) z^{k}
$$

we have

$$
\left[x^{r} y^{s}\right]\left\{(1-x)^{-(m-r-s+1)}(1-y)^{-(m-r-s+1)}\right\}=\left(\begin{array}{c}
m-s \\
r
\end{array}\right)\left(\begin{array}{c}
m-r \\
s
\end{array}\right),
$$

and

$$
\begin{gathered}
{\left[x^{r} y^{s}\right]\left\{(1-x)^{-(m-r-s+1)}(1-y)^{-(m-r-s+1)} x^{m+1} y^{m+1}\right\}} \\
\quad= \begin{cases}0, & \text { if } r \leq m \text { or } s \leq m, \\
(-1)^{r+s}\left(\begin{array}{c}
r+s-m-1 \\
s
\end{array}\right)\left(\begin{array}{c}
r+s-m-1 \\
r
\end{array}\right), & \text { if } r, s \geq m+1 .\end{cases}
\end{gathered}
$$

But, it is easy to see that

$$
\left(\begin{array}{c}
m-s \\
r
\end{array}\right)\left(\begin{array}{c}
m-r \\
s
\end{array}\right)=(-1)^{r+s}\left(\begin{array}{c}
r+s-m-1 \\
s
\end{array}\right)\left(\begin{array}{c}
r+s-m-1 \\
r
\end{array}\right) .
$$

Substituting these into (2.10) yields

$$
f(x, y)=\sum_{0 \leq r, s \leq m} u^{r} v^{s}\left(\begin{array}{c}
m-s \\
r
\end{array}\right)\left(\begin{array}{c}
m-r \\
s
\end{array}\right) .
$$

Corollary 2.5 For $m \in \mathbb{N}$, we have

$$
\begin{aligned}
& \sum_{r=0}^{m} \sum_{s=o}^{m-r}\left(\begin{array}{c}
m-r+1 \\
s
\end{array}\right)\left(\begin{array}{c}
m-s \\
r
\end{array}\right) \frac{x^{r} y^{s}}{(1-x)^{r+s}(1-y)^{r+s}} \\
& =\frac{1-x^{m+2} y^{m+2}-x\left(1-x^{m+1} y^{m+1}\right)-(1-x y) y^{m+1}}{(1-x y)(1-x)^{m+1}(1-y)^{m+1}} .
\end{aligned}
$$

Proof. Replacing $m$ and $r$ by $m-1$ and $r-1$ respectively in (2.8), we obtain

$$
\sum_{r=1}^{m} \sum_{s=o}^{m-r}\left(\begin{array}{c}
m-r \\
s
\end{array}\right)\left(\begin{array}{c}
m-s-1 \\
r-1
\end{array}\right) \frac{x^{r} y^{s}}{(1-x)^{r+s}(1-y)^{r+s}}=\frac{x\left(1-x^{m} y^{m}\right)}{(1-x y)(1-x)^{m}(1-y)^{m}} .
$$


Combining (2.8) and (2.12), we get

$$
\sum_{r=0}^{m} \sum_{s=0}^{m-r}\left(\begin{array}{c}
m-r \\
s
\end{array}\right)\left(\begin{array}{c}
m-s-1 \\
r
\end{array}\right) \frac{x^{r} y^{s}}{(1-x)^{r+s}(1-y)^{r+s}}=\frac{1-x^{m+1} y^{m+1}-x\left(1-x^{m} y^{m}\right)}{(1-x y)(1-x)^{m}(1-y)^{m}} .
$$

Replacing $m$ by $m+1$ in (2.13), we have

$$
\begin{aligned}
& \sum_{r=0}^{m+1} \sum_{s=o}^{m-r+1}\left(\begin{array}{c}
m-r+1 \\
s
\end{array}\right)\left(\begin{array}{c}
m-s \\
r
\end{array}\right) \frac{x^{r} y^{s}}{(1-x)^{r+s}(1-y)^{r+s}} \\
& \quad=\frac{1-x^{m+2} y^{m+2}-x\left(1-x^{m+1} y^{m+1}\right)}{(1-x y)(1-x)^{m+1}(1-y)^{m+1}} .
\end{aligned}
$$

Note that when $r=m+1,\left(\begin{array}{c}m-s \\ r\end{array}\right)=0$, and when $s=m-r+1,\left(\begin{array}{c}m-s \\ r\end{array}\right)=\left(\begin{array}{c}r-1 \\ r\end{array}\right)$ is equal to 1 if $r=0$ and 0 otherwise. Moving the term $\frac{y^{m+1}}{(1-x)^{m+1}(1-y)^{m+1}}$ of $(2.14)$ from the left-hand side to the right-hand side, we obtain (2.11).

Interchanging $r$ and $s$, and $x$ and $y$ in (2.11), we get

$$
\begin{gathered}
\sum_{r=0}^{m} \sum_{s=o}^{m-r}\left(\begin{array}{c}
m-r \\
s
\end{array}\right)\left(\begin{array}{c}
m-s+1 \\
r
\end{array}\right) \frac{x^{r} y^{s}}{(1-x)^{r+s}(1-y)^{r+s}} \\
=\frac{1-x^{m+2} y^{m+2}-y\left(1-x^{m+1} y^{m+1}\right)-(1-x y) x^{m+1}}{(1-x y)(1-x)^{m+1}(1-y)^{m+1}} .
\end{gathered}
$$

Corollary 2.6 For $m \in \mathbb{N}$, we have

$$
\frac{\left(1-x^{m+1}\right)\left(1-y^{m+1}\right)}{(1-x)^{m+1}(1-y)^{m+1}}=\sum_{r=0}^{m} \sum_{s=o}^{m-r} \frac{m+1}{m+1-r-s}\left(\begin{array}{c}
m-r \\
s
\end{array}\right)\left(\begin{array}{c}
m-s \\
r
\end{array}\right) \frac{x^{r} y^{s}}{(1-x)^{r+s}(1-y)^{r+s}} .
$$

Proof. Note that

$$
\begin{aligned}
& \frac{m+1}{m+1-r-s}\left(\begin{array}{c}
m-r \\
s
\end{array}\right)\left(\begin{array}{c}
m-s \\
r
\end{array}\right) \\
& =\left(\begin{array}{c}
m-r+1 \\
s
\end{array}\right)\left(\begin{array}{c}
m-s \\
r
\end{array}\right)+\left(\begin{array}{c}
m-r \\
s
\end{array}\right)\left(\begin{array}{c}
m-s+1 \\
r
\end{array}\right)-\left(\begin{array}{c}
m-r \\
s
\end{array}\right)\left(\begin{array}{c}
m-s \\
r
\end{array}\right) .
\end{aligned}
$$

Hence, from (2.8), (2.11) and (2.15) it follows that

$$
\begin{aligned}
\sum_{r=0}^{m} & \sum_{s=o}^{m-r} \frac{m+1}{m+1-r-s}\left(\begin{array}{c}
m-r \\
s
\end{array}\right)\left(\begin{array}{c}
m-s \\
r
\end{array}\right) \frac{x^{r} y^{s}}{(1-x)^{r+s}(1-y)^{r+s}} \\
= & \frac{2-2 x^{m+2} y^{m+2}-(x+y)\left(1-x^{m+1} y^{m+1}\right)-(1-x y)\left(x^{m+1}+y^{m+1}\right)}{(1-x y)(1-x)^{m+1}(1-y)^{m+1}} \\
& -\frac{1-x^{m+1} y^{m+1}}{(1-x y)(1-x)^{m}(1-y)^{m}} .
\end{aligned}
$$


After simplification, we obtain (2.16).

It is easy to see that (2.16) may be written as:

$$
\begin{aligned}
& \left(1-x^{m}\right)\left(1-y^{m}\right) \\
& =\sum_{k=0}^{m-1} \sum_{i=0}^{k} \frac{m}{m-k}\left(\begin{array}{c}
m-k+i-1 \\
i
\end{array}\right)\left(\begin{array}{c}
m-i-1 \\
k-i
\end{array}\right) x^{i} y^{k-i}(1-x)^{m-k}(1-y)^{m-k} .
\end{aligned}
$$

Remark. Applying the multivariate Lagrange inversion formula, we can also prove (2.11) and (2.16) as well as the following generalization of (2.8):

$$
\sum_{r_{1}, \ldots, r_{m} \leq n} \prod_{k=1}^{m}\left(\begin{array}{c}
n-r_{k} \\
r_{k+1}
\end{array}\right) \frac{x_{k}^{r_{k}}}{\left(1-x_{k}\right)^{r_{k}+r_{k+1}}}=\frac{1-(-1)^{m(n+1)} x_{1}^{n+1} \cdots x_{m}^{n+1}}{1-(-1)^{m} x_{1} \cdots x_{m}} \prod_{k=1}^{m} \frac{1}{\left(1-x_{k}\right)^{n}},
$$

where $r_{m+1}=r_{1}$.

Recall the Vandermonde determinant formula:

$$
\operatorname{det}\left(x_{i}^{n-j}\right)_{1 \leq i, j \leq n}=\prod_{1 \leq i<j \leq n}\left(x_{i}-x_{j}\right)
$$

Let $e_{i}\left(x_{1}, \ldots, x_{n}\right)(0 \leq i \leq n)$ be the $i$-th elementary symmetric function of $x_{1}, \ldots, x_{n}$, and let

$$
\left(x_{1}, \ldots, \hat{x}_{j}, \ldots, x_{n}\right)=\left(x_{1}, \ldots, x_{j-1}, x_{j+1}, \ldots, x_{n}\right), \quad 1 \leq j \leq n .
$$

Lemma 2.7 Let $A=\left(x_{i}^{n-j}\right)_{1 \leq i, j \leq n}$ be the Vandermonde matrix. Then

$$
A^{-1}=\left((-1)^{n-i} \frac{e_{i-1}\left(x_{1}, \ldots, \hat{x_{j}}, \ldots, x_{n}\right)}{\prod_{k=1, k \neq j}^{n}\left(x_{k}-x_{j}\right)}\right)_{1 \leq i, j \leq n} .
$$

Proof. The elementary symmetric functions satisfy the identity

$$
\sum_{k=0}^{n}(-t)^{n-k} e_{k}\left(x_{1}, \ldots, x_{n}\right)=\prod_{k=1}^{n}\left(x_{k}-t\right)
$$

Therefore, for each $j=1,2, \ldots, n$, we have

$$
\sum_{k=1}^{n}(-t)^{n-k} e_{k-1}\left(x_{1}, \ldots, \hat{x}_{j}, \ldots, x_{n}\right)=\prod_{\substack{k=1 \\ k \neq j}}^{n}\left(x_{k}-t\right) .
$$

The result then follows by setting $t=x_{i}(1 \leq i \leq n)$ in $(2.20)$.

We shall need the following variant of Vandermonde's determinant. 
Lemma 2.8 Let $A=\left(\left(1-x^{i}\right)^{n+1-j}\left(1-x^{i+1}\right)^{n+1-j} x^{i(j-1)}\right)_{1 \leq i, j \leq n}$. Then

$$
\operatorname{det} A=(-1)^{\frac{n(n-1)}{2}} x^{\frac{n\left(n^{2}-1\right)}{6}} \prod_{k=1}^{n}\left(1-x^{2 k-1}\right)^{n+1-k}\left(1-x^{2 k}\right)^{n+1-k} \text {. }
$$

Proof. Extracting $x^{(n-1) i}\left(1-x^{i}\right)\left(1-x^{i+1}\right)$ from the $i$-th row $(1 \leq i \leq n)$ of $A$ and then applying the Vandermonde determinant formula, we obtain

$$
\begin{aligned}
\operatorname{det} A & =\prod_{i=1}^{n} x^{(n-1) i}\left(1-x^{i}\right)\left(1-x^{i+1}\right) \cdot \operatorname{det}\left(\frac{\left(1-x^{i}\right)^{n-j}\left(1-x^{i+1}\right)^{n-j}}{x^{i(n-j)}}\right)_{1 \leq i, j \leq n} \\
& =\prod_{i=1}^{n} x^{(n-1) i}\left(1-x^{i}\right)\left(1-x^{i+1}\right) \cdot \prod_{1 \leq i<j \leq n}\left(\frac{\left(1-x^{i}\right)\left(1-x^{i+1}\right)}{x^{i}}-\frac{\left(1-x^{j}\right)\left(1-x^{j+1}\right)}{x^{j}}\right) \\
& =\prod_{i=1}^{n} x^{(n-1) i}\left(1-x^{i}\right)\left(1-x^{i+1}\right) \cdot \prod_{1 \leq i<j \leq n} \frac{-\left(1-x^{j-i}\right)\left(1-x^{i+j+1}\right)}{x^{j}}
\end{aligned}
$$

which yields the desired formula after simplification.

\section{Proof of Theorems}

Theorem 3.1 For any $m \in \mathbb{N}$, there exist polynomials $P_{m, k}(y) \in \mathbb{Z}[y]$ such that

$$
\begin{gathered}
\sum_{k=0}^{m}(-1)^{k}\left[\left(\begin{array}{c}
2 m \\
k
\end{array}\right)-\left(\begin{array}{c}
2 m \\
k-2
\end{array}\right)\right] \frac{\left(1-x^{m+1-k}\right)\left(1-x^{m+1-k} y^{m+1-k}\right) x^{k}}{1-y^{m+1-k}} \\
=\sum_{k=0}^{m}(-1)^{k} P_{m, k}(y) \frac{(1-x)^{m+1-k}(1-x y)^{m+1-k}(1-y)^{3 k} x^{k}}{\prod_{i=0}^{k}\left(1-y^{m+1-i}\right)} .
\end{gathered}
$$

Proof. By formula (2.18), we have

$$
\begin{aligned}
(1- & \left.x^{m+1-k}\right)\left(1-x^{m+1-k} y^{m+1-k}\right) x^{k} \\
= & \sum_{r=0}^{m-k} \sum_{i=0}^{r} \frac{m-k+1}{m-k-r+1}\left(\begin{array}{c}
m-k-r+i \\
i
\end{array}\right)\left(\begin{array}{c}
m-k-i \\
r-i
\end{array}\right) \\
& \times x^{k+r} y^{r-i}(1-x)^{m-k-r+1}(1-x y)^{m-k-r+1} .
\end{aligned}
$$

Therefore, setting $s=r+k$, we obtain

$$
\begin{aligned}
& \sum_{k=0}^{m} \frac{(-1)^{k}}{1-y^{m+1-k}}\left[\left(\begin{array}{c}
2 m \\
k
\end{array}\right)-\left(\begin{array}{c}
2 m \\
k-2
\end{array}\right)\right]\left(1-x^{m+1-k}\right)\left(1-x^{m+1-k} y^{m+1-k}\right) x^{k} \\
& \quad=\sum_{s=0}^{m} \bar{P}_{m, s}(y) \frac{(1-x)^{m+1-s}(1-x y)^{m+1-s} x^{s}}{\prod_{i=0}^{k}\left(1-y^{m+1-i}\right)}
\end{aligned}
$$


where $\bar{P}_{m, s}(y)$ are polynomials given by

$$
\begin{aligned}
\bar{P}_{m, s}(y)= & \sum_{k=0}^{s}(-1)^{k}\left[\left(\begin{array}{c}
2 m \\
k
\end{array}\right)-\left(\begin{array}{c}
2 m \\
k-2
\end{array}\right)\right] \prod_{\substack{j=0 \\
j \neq k}}^{s}\left(1-y^{m+1-j}\right) \\
& \times \sum_{i=0}^{s-k} \frac{m-k+1}{m-s+1}\left(\begin{array}{c}
m-s+i \\
i
\end{array}\right)\left(\begin{array}{c}
m-k-i \\
s-k-i
\end{array}\right) y^{s-k-i} .
\end{aligned}
$$

By (2.17), we have $\bar{P}_{m, s}(y) \in \mathbb{Z}[y]$. It remains to show that $(1-y)^{3 s} \mid \bar{P}_{m, s}(y)$.

In view of Lemma 2.1, setting $x=q^{n}$ and $y=q$ in (3.2), the left-hand side reduces to $\left(1-q^{2}\right)(1-q)^{2 m} S_{2 m+1, n}(q)$. Therefore, it follows from (1.1) and (3.2) that

$$
\sum_{k=0}^{m} \bar{P}_{m, k}(q) \frac{\left(1-q^{n}\right)^{m+1-k}\left(1-q^{n+1}\right)^{m+1-k} q^{k n}}{\prod_{i=0}^{k}\left(1-q^{m+1-i}\right)}=\sum_{k=1}^{n}\left(1-q^{2 k}\right)\left(1-q^{k}\right)^{2 m} q^{(m+1)(n-k)} .
$$

Taking $n=1,2, \ldots, m+1$ in (3.4), we obtain the following matrix equation

$$
A \cdot\left(\begin{array}{c}
x_{1} \\
x_{2} \\
\vdots \\
x_{m+1}
\end{array}\right)=\left(\begin{array}{c}
b_{1} \\
b_{2} \\
\vdots \\
b_{m+1}
\end{array}\right),
$$

where $A=\left(\left(1-q^{i}\right)\left(1-q^{i+1}\right) q^{m i} a_{i}^{m+1-j}\right)_{1 \leq i, j \leq m+1}$ with $a_{i}=\left(1-q^{i}\right)\left(1-q^{i+1}\right) / q^{i}$, and where

$$
\begin{aligned}
b_{i} & =\sum_{k=1}^{i}\left(1-q^{2 k}\right)\left(1-q^{k}\right)^{2 m} q^{(m+1)(i-k)}, \\
x_{j} & =\frac{\bar{P}_{m, j-1}(q)}{\prod_{k=0}^{j-1}\left(1-q^{m+1-k}\right)} .
\end{aligned}
$$

Now Lemma 2.8 implies that $\operatorname{det} A \neq 0$, so Equation (3.5) has a unique solution given by

$$
x_{j}=\sum_{i=1}^{m+1}\left(A^{-1}\right)_{j i} b_{i}, \quad j=1,2, \ldots, m+1,
$$

where, by Lemma 2.7,

$$
\begin{aligned}
\left(A^{-1}\right)_{j i} & =\frac{(-1)^{m+1-j}}{q^{m i}\left(1-q^{i}\right)\left(1-q^{i+1}\right)} \frac{e_{j-1}\left(a_{1}, \ldots, \hat{a_{i}}, \ldots, a_{m+1}\right)}{\prod_{k=1, k \neq i}^{m+1}\left(a_{k}-a_{i}\right)} \\
& =\frac{(-1)^{m+i-j} q^{\left({ }_{2}^{m+2-i}\right)}\left(1-q^{2 i+1}\right) e_{j-1}\left(a_{1}, \ldots, \hat{a}_{i}, \ldots, a_{m+1}\right)}{(q ; q)_{m+i+2}(q ; q)_{m-i+1}} .
\end{aligned}
$$


Here we have adopted the notation $(q ; q)_{n}=(1-q)\left(1-q^{2}\right) \cdots\left(1-q^{n}\right)$. It follows from (3.6) and (3.7) that

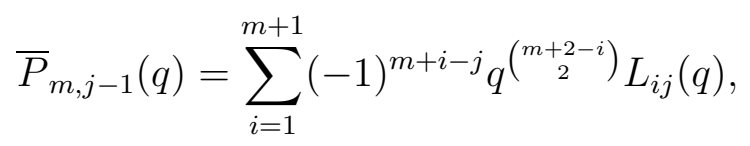

where

$$
L_{i j}(q)=\frac{\left(1-q^{2 i+1}\right) e_{j-1}\left(a_{1}, \ldots, \hat{a}_{i}, \ldots, a_{m+1}\right)(q ; q)_{m+1} b_{i}}{(q ; q)_{m+i+2}(q ; q)_{m-i+1}(q ; q)_{m-j+1}} .
$$

Since $a_{i}=\left(1-q^{i}\right)\left(1-q^{i+1}\right) / q^{i}$, the valuation of $(1-q)$ in $e_{j-1}\left(a_{1}, \ldots, \hat{a}_{i}, \ldots, a_{m+1}\right)$ is at least $2 j-2$. Also, it is clear that $(1-q)^{2 m+1} \mid b_{i}$ for $i=1,2, \ldots, m+1$. Hence, from (3.8) it follows that the valuation of $(1-q)$ in $L_{i j}(q)$ is at least $3 j-3$ for $j=1,2, \ldots, m+1$. Therefore, the polynomials

$$
P_{m, j}(q)=(-1)^{j} \frac{\bar{P}_{m, j}(q)}{(1-q)^{3 j}}, \quad j=0,1, \ldots, m,
$$

satisfy (3.1). This completes the proof.

The proof of Theorem 1.1 then follows from (3.4) and (3.9).

Multiplying $(2.8)$ by $(1-x)^{m}(1-y)^{m}$ and putting $r=i$ and $s=k-i$, we get

$$
\frac{1-x^{m+1} y^{m+1}}{1-x y}=\sum_{k=0}^{m} \sum_{i=0}^{k}\left(\begin{array}{c}
m-k+i \\
i
\end{array}\right)\left(\begin{array}{c}
m-i \\
k-i
\end{array}\right) x^{i} y^{k-i}(1-x)^{m-k}(1-y)^{m-k} .
$$

Writing

$$
\frac{1-x^{2 m+1} y^{2 m+1}}{1-x y}=\frac{1-x^{2 m+2} y^{2 m+2}}{1-x^{2} y^{2}}+x y \frac{1-x^{2 m} y^{2 m}}{1-x^{2} y^{2}}
$$

we obtain

$$
\begin{aligned}
& \frac{1-x^{2 m+1} y^{2 m+1}}{1-x y} \\
& =\sum_{k=0}^{m} x^{k} \sum_{i=0}^{k}\left(\begin{array}{c}
m-k+i \\
i
\end{array}\right)\left(\begin{array}{c}
m-i \\
k-i
\end{array}\right) y^{2 k-2 i}(1-x)^{m-k}\left(1-x y^{2}\right)^{m-k} \\
& \quad+\sum_{k=1}^{m} x^{k} \sum_{i=0}^{k-1}\left(\begin{array}{c}
m-k+i \\
i
\end{array}\right)\left(\begin{array}{c}
m-i-1 \\
k-i-1
\end{array}\right) y^{2 k-2 i-1}(1-x)^{m-k}\left(1-x y^{2}\right)^{m-k} .
\end{aligned}
$$

Theorem 3.2 For any $m \in \mathbb{N}$, there exist polynomials $Q_{m, k}(y) \in \mathbb{Z}[y]$ such that

$$
\begin{gathered}
\sum_{k=0}^{m}(-1)^{k}\left[\left(\begin{array}{c}
2 m-1 \\
k
\end{array}\right)-\left(\begin{array}{c}
2 m-1 \\
k-2
\end{array}\right)\right] \frac{\left(1-x^{2 m-2 k+1} y^{2 m-2 k+1}\right) x^{k}}{1-y^{2 m-2 k+1}} \\
=\sum_{k=0}^{m}(-1)^{k} Q_{m, k}(y) \frac{(1-x)^{m-k}\left(1-x y^{2}\right)^{m-k}(1-x y)(1-y)^{3 k} x^{k}}{\prod_{i=0}^{k}\left(1-y^{2 m-2 i+1}\right)} .
\end{gathered}
$$


Proof. By formula (3.10), we have

$$
\begin{aligned}
(1- & \left.x^{2 m-2 k+1} y^{2 m-2 k+1}\right) x^{k} \\
= & \sum_{r=0}^{m-k} \sum_{i=0}^{r}\left(\begin{array}{c}
m-k-r+i \\
i
\end{array}\right)\left[\left(\begin{array}{c}
m-k-i \\
r-i
\end{array}\right) y^{2 r-2 i}+\left(\begin{array}{c}
m-k-i-1 \\
r-i-1
\end{array}\right) y^{2 r-2 i-1}\right] \\
& \times x^{k+r}(1-x)^{m-k-r}\left(1-x y^{2}\right)^{m-k-r}(1-x y) .
\end{aligned}
$$

Therefore, setting $s=r+k$, we get

$$
\begin{aligned}
& \sum_{k=0}^{m}(-1)^{k}\left[\left(\begin{array}{c}
2 m-1 \\
k
\end{array}\right)-\left(\begin{array}{c}
2 m-1 \\
k-2
\end{array}\right)\right] \frac{\left(1-x^{2 m-2 k+1} y^{2 m-2 k+1}\right) x^{k}}{1-y^{2 m-2 k+1}} \\
& \quad=\sum_{s=0}^{m} \bar{Q}_{m, s}(y) \frac{x^{s}(1-x)^{m-s}\left(1-x y^{2}\right)^{m-s}(1-x y)}{\prod_{i=1}^{k}\left(1-y^{2 m-2 i+1}\right)}
\end{aligned}
$$

where

$$
\begin{aligned}
\bar{Q}_{m, s}(y)= & \sum_{k=0}^{s}(-1)^{k}\left[\left(\begin{array}{c}
2 m-1 \\
k
\end{array}\right)-\left(\begin{array}{c}
2 m-1 \\
k-2
\end{array}\right)\right] \prod_{\substack{j=0 \\
j \neq k}}^{s}\left(1-y^{2 m-2 j+1}\right) \sum_{i=0}^{s-k}\left(\begin{array}{c}
m-s+i \\
i
\end{array}\right) \\
& \times\left[\left(\begin{array}{c}
m-k-i \\
s-k-i
\end{array}\right) y^{2 s-2 k-2 i}+\left(\begin{array}{c}
m-k-i-1 \\
s-k-i-1
\end{array}\right) y^{2 s-2 k-2 i-1}\right] .
\end{aligned}
$$

What remains is to show that

$$
(1-q)^{s}\left(1-q^{2}\right)^{2 s} \mid \bar{Q}_{m, s}(q) \text { and } Q_{m, s}(q)=(-1)^{s} \frac{\bar{Q}_{m, s}(q)}{(1-q)^{s}\left(1-q^{2}\right)^{2 s}} .
$$

The proof is exactly the same as that of Theorem 3.1 and is omitted.

The proof of Theorem 1.2 then follows from (2.3) and (3.11) (replacing $x$ and $y$ by $q^{n}$ and $q^{\frac{1}{2}}$, respectively).

Remark. It follows from (1.7) and (1.8) that $P_{m, m}(q)=Q_{m, m}(q)=0$ if $m \geq 1$, and $P_{m, s}(0)=Q_{m, s}(0)=\left(\begin{array}{c}m+s-2 \\ s\end{array}\right)-\left(\begin{array}{c}m+s-2 \\ s-2\end{array}\right)$. Moreover, if $s<m$, the polynomial $P_{m, s}(q)$ has degree $s(2 m-3-s) / 2$ while $Q_{m, s}(q)$ has degree $s(2 m-3-s)$.

Proof of Theorem 1.3. By formula (2.18), we have

$$
\begin{aligned}
\left(1-x^{m-r}\right)\left(1-x^{m-r} y^{m-r}\right) x^{r}= & \sum_{k=0}^{m-r} \sum_{i=0}^{k} \frac{m-r}{m-r-k}\left(\begin{array}{c}
m-r-k+i-1 \\
i
\end{array}\right)\left(\begin{array}{c}
m-r-i-1 \\
k-i
\end{array}\right) \\
& \times x^{r+k} y^{k-i}(1-x)^{m-r-k}(1-x y)^{m-r-k} .
\end{aligned}
$$


Therefore, setting $s=r+k$ and

$$
\begin{aligned}
& \bar{G}_{m, s}(y) \\
& =\sum_{r=0}^{s}(-1)^{r}\left(\begin{array}{c}
2 m \\
r
\end{array}\right) \prod_{\substack{j=0 \\
j \neq r}}^{s}\left(1+y^{m-j}\right) \sum_{i=0}^{s-r} \frac{m-r}{m-s}\left(\begin{array}{c}
m-s+i-1 \\
i
\end{array}\right)\left(\begin{array}{c}
m-r-i-1 \\
s-r-i
\end{array}\right) y^{s-r-i},
\end{aligned}
$$

we obtain

$$
\sum_{r=0}^{m-1}(-1)^{r}\left(\begin{array}{c}
2 m \\
r
\end{array}\right) \frac{\left(1-x^{m-r}\right)\left(1-x^{m-r} y^{m-r}\right) x^{r}}{1+y^{m-r}}=\sum_{s=0}^{m} \bar{G}_{m, s}(y) \frac{(1-x)^{m-s}(1-x y)^{m-s} x^{s}}{\prod_{i=0}^{s}\left(1+y^{m-i}\right)} .
$$

Similarly to the proof of Theorem 3.1, we can show that $G_{m, s}(y)=(-1)^{s} \bar{G}_{m, s}(y) /(1-y)^{2 s}$ is a polynomial in $\mathbb{Z}[y]$.

Theorem 1.3 then follows from Lemma 2.2 after substituting $x=q^{n}$ and $y=q$ into (3.13).

The proof of Theorem 1.4 is analogous to that of Theorem 1.2 and is omitted here.

\section{Sums of $m$-th Powers for $m \leq 11$}

Theorems 1.1-1.4 permit us to compute $P_{m, k}(q), Q_{m, k}(q), G_{m, k}(q)$, and $H_{m, k}(q)$ quickly by using Maple. Tables 1-4 give the first values of these polynomials.

Table 1: Values of $P_{m, k}(q)$ for $0 \leq m \leq 5$.

\begin{tabular}{|l|r|r|r|r|r|r|}
\hline$k \backslash m$ & 0 & 1 & 2 & 3 & 4 & 5 \\
\hline 0 & 1 & 1 & 1 & 1 & 1 & 1 \\
\hline 1 & & & 1 & $2(q+1)$ & $3 q^{2}+4 q+3$ & $2(q+1)\left(2 q^{2}+q+2\right)$ \\
\hline 2 & & & & $2(q+1)$ & $(q+1)\left(5 q^{2}+8 q+5\right)$ & $(q+1)\left(9 q^{4}+19 q^{3}+29 q^{2}+19 q+9\right)$ \\
\hline 3 & & & & & $(q+1)\left(5 q^{2}+8 q+5\right)$ & $2(q+1)^{2}\left(q^{2}+q+1\right)\left(7 q^{2}+11 q+7\right)$ \\
\hline 4 & & & & & & $2(q+1)^{2}\left(q^{2}+q+1\right)\left(7 q^{2}+11 q+7\right)$ \\
\hline
\end{tabular}

Table 2: Values of $Q_{m, k}(q)$ for $1 \leq m \leq 4$

\begin{tabular}{|l|r|r|r|r|}
\hline$k \backslash m$ & 1 & 2 & 3 & 4 \\
\hline 0 & 1 & 1 & 1 & 1 \\
\hline 1 & & 1 & $2 q^{2}+q+2$ & $3 q^{4}+2 q^{3}+4 q^{2}+2 q+3$ \\
\hline 2 & & & $2 q^{2}+q+2$ & $\left(q^{2}+q+1\right)\left(5 q^{4}+q^{3}+9 q^{2}+q+5\right)$ \\
\hline 3 & & & & $\left(q^{2}+q+1\right)\left(5 q^{4}+q^{3}+9 q^{2}+q+5\right)$ \\
\hline
\end{tabular}


For $m=5$, we have $Q_{5,0}(q)=1$, and

$$
\begin{aligned}
Q_{5,1}(q) & =4 q^{6}+3 q^{5}+6 q^{4}+4 q^{3}+6 q^{2}+3 q+4, \\
Q_{5,2}(q) & =9 q^{10}+13 q^{9}+33 q^{8}+37 q^{7}+61 q^{6}+51 q^{5}+61 q^{4}+37 q^{3}+33 q^{2}+13 q+9, \\
Q_{5,3}(q) & =Q_{5,4}(q) \\
& =\left(q^{2}+q+1\right)\left(14 q^{10}+14 q^{9}+56 q^{8}+46 q^{7}+100 q^{6}+65 q^{5}+100 q^{4}+46 q^{3}+56 q^{2}+14 q+14\right) .
\end{aligned}
$$

Table 3: Values of $G_{m, k}(q)$ for $1 \leq m \leq 5$.

\begin{tabular}{|l|r|r|r|r|r|}
\hline$k \backslash m$ & 1 & 2 & 3 & 4 & 5 \\
\hline 0 & 1 & 1 & 1 & 1 & 1 \\
\hline 1 & & 2 & $3(q+1)$ & $4\left(q^{2}+q+1\right)$ & $5(q+1)\left(q^{2}+1\right)$ \\
\hline 2 & & & $6(q+1)$ & $2(q+1)\left(5 q^{2}+7 q+5\right)$ & $5(q+1)\left(3 q^{4}+4 q^{3}+8 q^{2}+4 q+3\right)$ \\
\hline 3 & & & & $4(q+1)\left(5 q^{2}+7 q+5\right)$ & $5(q+1)^{2}\left(7 q^{4}+14 q^{3}+20 q^{2}+14 q+7\right)$ \\
\hline 4 & & & & & $10(q+1)^{2}\left(7 q^{4}+14 q^{3}+20 q^{2}+14 q+7\right)$ \\
\hline
\end{tabular}

Table 4: Values of $H_{m, k}(q)$ for $1 \leq m \leq 4$.

\begin{tabular}{|l|r|r|r|r|}
\hline$k \backslash m$ & 1 & 2 & 3 & 4 \\
\hline 0 & 1 & 1 & 1 & 1 \\
\hline 1 & & 2 & $3 q^{2}+2 q+3$ & $4 q^{4}+3 q^{3}+4 q^{2}+3 q+4$ \\
\hline 2 & & & $2\left(3 q^{2}+2 q+3\right)$ & $10 q^{6}+15 q^{5}+30 q^{4}+26 q^{3}+30 q^{2}+15 q+10$ \\
\hline 3 & & & & $2\left(10 q^{6}+15 q^{5}+30 q^{4}+26 q^{3}+30 q^{2}+15 q+10\right)$ \\
\hline
\end{tabular}

Substituting the values of Tables 1 and 2 into Theorems 1.1 and 1.2 yields the summation formulas for sums of $m$-th power for $m=1,2, \ldots, 11$. In particular, for $1 \leq m \leq 5$ we recover the formulas (1.2)-(1.6) of Warnaar and Schlosser. For $m=6,7, \ldots, 11$ we obtain the following formulas of Faulhaber type:

$$
\begin{aligned}
S_{6, n}(q)= & \frac{\left(1-q^{n}\right)\left(1-q^{n+1}\right)\left(1-q^{n+\frac{1}{2}}\right)}{(1-q)\left(1-q^{2}\right)\left(1-q^{\frac{7}{2}}\right)}\left[\frac{\left(1-q^{n}\right)^{2}\left(1-q^{n+1}\right)^{2}}{(1-q)^{4}}\right. \\
& \left.-\left(2+2 q+q^{\frac{1}{2}}\right)\left(\frac{\left(1-q^{n}\right)\left(1-q^{n+1}\right) q^{n}}{\left(1+q^{\frac{1}{2}}\right)(1-q)\left(1-q^{\frac{5}{2}}\right)}-\frac{\left(1-q^{\frac{1}{2}}\right)^{2} q^{2 n}}{\left(1-q^{\frac{3}{2}}\right)\left(1-q^{\frac{5}{2}}\right)}\right)\right], \\
S_{7, n}(q)= & \frac{\left(1-q^{n}\right)^{2}\left(1-q^{n+1}\right)^{2}}{(1-q)^{3}\left(1-q^{4}\right)} \\
& \times\left[\frac{\left(1-q^{n}\right)^{2}\left(1-q^{n+1}\right)^{2}}{(1-q)^{3}\left(1-q^{2}\right)}-\frac{2\left(1-q^{n}\right)\left(1-q^{n+1}\right) q^{n}}{(1-q)\left(1-q^{3}\right)}+\frac{2(1-q)^{2} q^{2 n}}{\left(1-q^{2}\right)\left(1-q^{3}\right)}\right],
\end{aligned}
$$




$$
\begin{aligned}
S_{8, n}(q)= & \frac{\left(1-q^{n}\right)\left(1-q^{n+1}\right)\left(1-q^{n+\frac{1}{2}}\right)}{(1-q)\left(1-q^{2}\right)\left(1-q^{\frac{9}{2}}\right)} \\
& \times\left[\frac{\left(1-q^{n}\right)^{3}\left(1-q^{n+1}\right)^{3}}{(1-q)^{6}}-\left(3+2 q^{\frac{1}{2}}+4 q+2 q^{\frac{3}{2}}+3 q^{2}\right) \frac{\left(1-q^{n}\right)^{2}\left(1-q^{n+1}\right)^{2} q^{n}}{\left(1+q^{\frac{1}{2}}\right)(1-q)^{3}\left(1-q^{\frac{7}{2}}\right)}\right. \\
& +\left(5+q^{\frac{1}{2}}+9 q+q^{\frac{3}{2}}+5 q^{2}\right) \frac{\left(1-q^{\frac{3}{2}}\right)\left(1-q^{n}\right)\left(1-q^{n+1}\right) q^{2 n}}{\left(1+q^{\frac{1}{2}}\right)(1-q)\left(1-q^{\frac{5}{2}}\right)\left(1-q^{\frac{7}{2}}\right)} \\
& \left.-\left(5+q^{\frac{1}{2}}+9 q+q^{\frac{3}{2}}+5 q^{2}\right) \frac{\left(1-q^{\frac{1}{2}}\right)^{3} q^{3 n}}{\left(1-q^{\frac{5}{2}}\right)\left(1-q^{\frac{7}{2}}\right)}\right], \\
S_{9, n}(q)= & \frac{\left(1-q^{n}\right)^{2}\left(1-q^{n+1}\right)^{2}}{(1-q)^{3}\left(1-q^{5}\right)} \\
& \times\left[\frac{\left(1-q^{n}\right)^{3}\left(1-q^{n+1}\right)^{3}}{(1-q)^{5}\left(1-q^{2}\right)}-\frac{\left(3 q^{2}+4 q+3\right)\left(1-q^{n}\right)^{2}\left(1-q^{n+1}\right)^{2} q^{n}}{(1-q)^{2}\left(1-q^{2}\right)\left(1-q^{4}\right)}\right. \\
& \left.+\left(5 q^{2}+8 q+5\right)\left(\frac{\left(1-q^{n}\right)\left(1-q^{n+1}\right) q^{2 n}}{\left(1-q^{3}\right)\left(1-q^{4}\right)}-\frac{(1-q)^{3} q^{3 n}}{\left(1-q^{2}\right)\left(1-q^{3}\right)\left(1-q^{4}\right)}\right)\right],
\end{aligned}
$$

$S_{10, n}(q)$

$$
\begin{aligned}
= & \frac{\left(1-q^{n}\right)\left(1-q^{n+1}\right)\left(1-q^{n+\frac{1}{2}}\right)}{(1-q)\left(1-q^{2}\right)\left(1-q^{\frac{11}{2}}\right)}\left[\frac{\left(1-q^{n}\right)^{4}\left(1-q^{n+1}\right)^{4}}{(1-q)^{8}}\right. \\
& -Q_{5,1}\left(q^{\frac{1}{2}}\right) \frac{\left(1-q^{\frac{1}{2}}\right)\left(1-q^{n}\right)^{3}\left(1-q^{n+1}\right)^{3} q^{n}}{(1-q)^{6}\left(1-q^{\frac{9}{2}}\right)}+Q_{5,2}\left(q^{\frac{1}{2}}\right) \frac{\left(1-q^{\frac{1}{2}}\right)^{2}\left(1-q^{n}\right)^{2}\left(1-q^{n+1}\right)^{2} q^{2 n}}{(1-q)^{4}\left(1-q^{\frac{7}{2}}\right)\left(1-q^{\frac{9}{2}}\right)} \\
& \left.-Q_{5,3}\left(q^{\frac{1}{2}}\right) \frac{\left(1-q^{\frac{1}{2}}\right)^{3}\left(1-q^{n}\right)\left(1-q^{n+1}\right) q^{3 n}}{(1-q)^{2}\left(1-q^{\frac{5}{2}}\right)\left(1-q^{\frac{7}{2}}\right)\left(1-q^{\frac{9}{2}}\right)}+\frac{Q_{5,4}\left(q^{\frac{1}{2}}\right)\left(1-q^{\frac{1}{2}}\right)^{4} q^{4 n}}{\left(1-q^{\frac{3}{2}}\right)\left(1-q^{\frac{5}{2}}\right)\left(1-q^{\frac{7}{2}}\right)\left(1-q^{\frac{9}{2}}\right)}\right],
\end{aligned}
$$

$S_{11, n}(q)$

$$
\begin{aligned}
= & \frac{\left(1-q^{n}\right)^{2}\left(1-q^{n+1}\right)^{2}}{(1-q)^{3}\left(1-q^{6}\right)}\left[\frac{\left(1-q^{n}\right)^{4}\left(1-q^{n+1}\right)^{4}}{(1-q)^{7}\left(1-q^{2}\right)}-\frac{2\left(2 q^{2}+q+2\right)\left(1-q^{n}\right)^{3}\left(1-q^{n+1}\right)^{3} q^{n}}{(1-q)^{5}\left(1-q^{5}\right)}\right. \\
& +\frac{\left(9 q^{4}+19 q^{3}+29 q^{2}+19 q+9\right)\left(1-q^{n}\right)^{2}\left(1-q^{n+1}\right)^{2} q^{2 n}}{(1-q)^{2}\left(1-q^{4}\right)\left(1-q^{5}\right)} \\
& \left.-2(q+1)\left(7 q^{2}+11 q+7\right)\left(\frac{\left(1-q^{n}\right)\left(1-q^{n+1}\right) q^{3 n}}{\left(1-q^{4}\right)\left(1-q^{5}\right)}-\frac{(1-q)^{3} q^{4 n}}{\left(1-q^{2}\right)\left(1-q^{4}\right)\left(1-q^{5}\right)}\right)\right] .
\end{aligned}
$$


From the computational point of view, with the help of Maple or other softwares, it is, of course, not difficult to give further extension of the above list of $S_{m, n}(q)$ 's.

\section{$5 \quad$ Further Remarks}

For $r \in \mathbb{N}$, define the following more general summation

$$
S_{m, n, r}(q)=\sum_{k=1}^{n} \frac{1-q^{(2 r+2) k}}{1-q^{2 r+2}}\left(\frac{1-q^{k}}{1-q}\right)^{m-1} q^{\frac{m+2 r+1}{2}(n-k)} .
$$

Then we can also obtain a similar summation formula.

Theorem 5.1 For $0 \leq r \leq m$, there exist polynomials $P_{m, k, r}(q) \in \mathbb{Z}[q]$ such that

$$
S_{2 m-2 r+1, n, r}(q)=\sum_{k=0}^{m}(-1)^{k} P_{m, k, r}(q) \frac{\left(1-q^{n}\right)^{m+1-k}\left(1-q^{n+1}\right)^{m+1-k} q^{k n}}{\left(1-q^{2 r+2}\right)(1-q)^{2 m-3 k} \prod_{i=0}^{k}\left(1-q^{m+1-i}\right)} .
$$

Furthermore, we have

$$
\begin{aligned}
P_{m, s, r}(q)= & \frac{\prod_{j=0}^{s}\left(1-q^{m+1-j}\right)}{(1-q)^{3 s-2 r}} \sum_{k=0}^{s} \frac{(-1)^{s-k}}{1-q^{m+1-k}}\left[\left(\begin{array}{c}
2 m-2 r \\
k
\end{array}\right)-\left(\begin{array}{c}
2 m-2 r \\
k-2 r-2
\end{array}\right)\right] \\
& \times \sum_{i=0}^{s-k} \frac{m-k+1}{m-s+1}\left(\begin{array}{c}
m-s+i \\
i
\end{array}\right)\left(\begin{array}{c}
m-k-i \\
s-k-i
\end{array}\right) q^{s-k-i} .
\end{aligned}
$$

Theorem 5.2 For $0 \leq r \leq m$, there exist polynomials $Q_{m, k, r}(q) \in \mathbb{Z}[q]$ such that

$$
S_{2 m-2 r, n, r}(q)=\sum_{k=0}^{m}(-1)^{k} Q_{m, k, r}\left(q^{\frac{1}{2}}\right) \frac{\left(1-q^{n+\frac{1}{2}}\right)\left(1-q^{n}\right)^{m-k}\left(1-q^{n+1}\right)^{m-k}\left(1-q^{\frac{1}{2}}\right)^{k} q^{k n}}{\left(1-q^{2 r+2}\right)(1-q)^{2 m-2 k-1} \prod_{i=0}^{k}\left(1-q^{m-i+\frac{1}{2}}\right)} .
$$

Furthermore, we have

$$
\begin{aligned}
Q_{m, s, r}(q)= & \frac{\prod_{j=0}^{s}\left(1-q^{2 m-2 j+1}\right)}{(1-q)^{s}\left(1-q^{2}\right)^{2 s-2 r}} \sum_{k=0}^{s} \frac{(-1)^{s-k}}{1-q^{2 m-2 k+1}}\left[\left(\begin{array}{c}
2 m-2 r-1 \\
k
\end{array}\right)-\left(\begin{array}{c}
2 m-2 r-1 \\
k-2 r-2
\end{array}\right)\right] \\
& \times \sum_{i=0}^{s-k}\left(\begin{array}{c}
m-s+i \\
i
\end{array}\right)\left[\left(\begin{array}{c}
m-k-i \\
s-k-i
\end{array}\right) q^{2 s-2 k-2 i}+\left(\begin{array}{c}
m-k-i-1 \\
s-k-i-1
\end{array}\right) q^{2 s-2 k-2 i-1}\right] .
\end{aligned}
$$

For example, we have

$$
\begin{aligned}
S_{4, n, 1}(q)= & \frac{\left(1-q^{n}\right)^{3}\left(1-q^{n+1}\right)^{3}\left(1-q^{n+\frac{1}{2}}\right)}{(1-q)^{3}\left(1-q^{4}\right)\left(1-q^{\frac{7}{2}}\right)}+\frac{\left(1+4 q^{\frac{1}{2}}+4 q+4 q^{\frac{3}{2}}+q^{2}\right)\left(1-q^{\frac{1}{2}}\right)}{1-q} \\
& \times \frac{\left(1-q^{n}\right)\left(1-q^{n+1}\right)\left(1-q^{n+\frac{1}{2}}\right)}{\left(1-q^{\frac{5}{2}}\right)\left(1-q^{\frac{7}{2}}\right)\left(1-q^{4}\right)}\left[\frac{\left(1-q^{n}\right)\left(1-q^{n+1}\right)}{(1-q)^{2}} q^{n+\frac{1}{2}}-\frac{1-q^{\frac{1}{2}}}{1-q^{\frac{3}{2}}} q^{2 n+\frac{1}{2}}\right],
\end{aligned}
$$


and

$$
\begin{aligned}
S_{5, n, 1}(q)= & \frac{\left(1-q^{n}\right)^{4}\left(1-q^{n+1}\right)^{4}}{(1-q)^{4}\left(1-q^{4}\right)^{2}} \\
& +\frac{4\left(1-q^{n}\right)^{2}\left(1-q^{n+1}\right)^{2}}{(1-q)\left(1-q^{3}\right)\left(1-q^{4}\right)^{2}}\left[\frac{(1+q)\left(1-q^{n}\right)\left(1-q^{n+1}\right)}{(1-q)^{2}} q^{n+1}-q^{2 n+1}\right] .
\end{aligned}
$$

There is a similar formula for

$$
T_{m, n, r}(q)=\sum_{k=1}^{n}(-1)^{n-k} \frac{1-q^{(2 r+1) k}}{1-q^{2 r+1}}\left(\frac{1-q^{k}}{1-q}\right)^{m-1} q^{\frac{m+2 r}{2}(n-k)},
$$

which is left to the interested readers.

In a forthcoming paper [6], it will be shown that the coefficients of the polynomials $P_{m, k}(q), Q_{m, k}(q), G_{m, k}(q)$ and $H_{m, k}(q)$ are actually nonnegative integers and have interesting combinatorial interpretations in terms of nonintersecting lattice paths.

Acknowledgment. The second author was supported by EC's IHRP Programme, within Research Training Network "Algebraic Combinatorics in Europe," grant HPRN-CT-200100272 .

\section{References}

[1] J. Faulhaber, Academia Algebra, Darinnen die miraculosische Inventiones zu den höchsten Cossen weiters continuirt und profitiert werden, Augspurg, bey Johann Ulrich Schönigs, 1631.

[2] K. C. Garrett and K. Hummel, A combinatorial proof of the sum of $q$-cubes, Electron. J. Combin. 11 (2004), \#R9.

[3] G. Gasper and M. Rahman, Basic Hypergeometric Series, Encyclopedia of Mathematics and Its Applications, Vol. 96, Second Edition, Cambridge University Press, Cambridge, 2004.

[4] I. M. Gessel and G. Viennot, Determinants, paths, and plane partitions, preprint, 1989.

[5] I. P. Goulden and D. M. Jackson, Combinatorial Enumeration, reprint of the 1983 original, Dover Publications, Inc., Mineola, NY, 2004.

[6] V. J. W. Guo, M. Rubey, and J. Zeng, Combinatorial interpretations of the $q$-Faulhaber and $q$-Salié coefficients, preprint, arXiv: math.CO/0506274.

[7] D. E. Knuth, Johann Faulhaber and sums of powers, Math. Comput. 61 (1993), 277-294.

[8] M. Schlosser, $q$-Analogues of the sums of consecutive integers, squares, cubes, quarts and quints, Electron. J. Combin. 11 (2004), \#R71.

[9] S. O. Warnaar, On the q-analogue of the sum of cubes, Electron. J. Combin. 11 (2004), \#N13. 